\title{
Pratiques
}

Linguistique, littérature, didactique

\section{La notion de tradition discursive : une perspective diachronique sur les genres textuels et sur les phénomènes de fréquence textuelle}

Sylvain Loiseau

\section{OpenEdition}

Journals

Édition électronique

URL : http://journals.openedition.org/pratiques/3731

DOI : 10.4000/pratiques.3731

ISSN : 2425-2042

Éditeur

Centre de recherche sur les médiations (CREM)

\section{Édition imprimée}

Date de publication : 1 juin 2013

Pagination : 91-104

Référence électronique

Sylvain Loiseau, «La notion de tradition discursive : une perspective diachronique sur les genres textuels et sur les phénomènes de fréquence textuelle », Pratiques [En ligne], 157-158 | 2013, mis en ligne le 18 décembre 2017, consulté le 07 mai 2019. URL : http://journals.openedition.org/ pratiques/3731; DOI : 10.4000/pratiques.3731 


\title{
La notion de tradition discursive : une perspective diachronique sur les genres textuels et sur les phénomènes de fréquence textuelle
}

\author{
Sylvain Loiseau
}

Laboratoire Lexiques, Dictionnaires, Informatique

(UMR 7187 CNRS/Université Paris Nord, Sorbonne Paris Cité)

\section{Introduction}

Cet article présente la notion de «tradition discursive». Cette notion, élaborée en Allemagne dans la tradition romaniste et particulièrement dans le cadre théorique d'Eugénio Coseriu est une contribution à la question des genres textuels encore peu connue en France.

De nombreuses notions ont été proposées pour rendre compte de l'appartenance des textes à des schémas plus abstraits qui expliquent leurs propriétés communes et la variation des contrats de réception et d'interprétation. L'originalité de la notion de tradition discursive est d'avoir été développée dans une perspective diachronique et de mettre l'accent sur l'historicité des textes. Dans cette perspective, ce qui explique particulièrement l'existence de propriétés communes entre des textes, c'est le fait que ces textes empruntent à des textes antérieurs des moules ou des séquences préfabriquées ; ces relations de répétitions et d'emprunts donnent lieu à des «traditions », des lignées de textes.

L'intérêt de cette notion est double. D'une part, elle permet de problématiser d'autres notions proches (genres, discours, type de texte...) en apportant un éclairage nouveau, celui de la diachronie.

D'autre part, les critères définitoires de cette notion, et notamment l'importance accordée à la répétition et à la traditionnalité, sont particulièrement intéressants pour l'interprétation des faits de fréquence textuelle qui intéresse l'analyse des genres au moyen de corpus textuels.

Je commencerai en présentant la notion de tradition discursive. J'essayerai ensuite de la situer parmi d'autres notions qui ont été proposées pour rendre compte des proximités entre textes. Les différentes propositions qui rendent compte de l'existence de grands schémas textuels mettent chacune l'accent sur un «principe typologique » différent. Elles peuvent mobiliser, par exemple, l'existence de pro- 
priétés formelles communes entre des groupes de textes, ou bien des propriétés praxéologiques différentes, etc. Enfin je soulignerai l'actualité et l'enjeu de la notion de tradition discursive pour l'interprétation des faits de fréquence.

\section{Les traditions discursives : une présentation}

La notion de Tradition discursive a été introduite par B. Schlieben-Lange (1983) et développée par des auteurs comme P. Koch (1997, 2008), H. Aschenberg (2003), R. Wilhelm (2001) et J. Kabatek (2001, 2011). Cette notion a été développée dans le cadre de la romanistique allemande et particulièrement à partir du cadre théorique d'Eugénio Coseriu. Elle s'inscrit donc dans un cadre variationnel plus vaste et dans une perspective théorique qui accorde une importance primordiale au phénomène de changement et à l'histoire. Cette notion est également caractérisée, outre ce contexte théorique général, par le fait qu'elle a été élaborée pour aider à la description de phénomènes concrets de changement - par exemple, rendre compte du fait que les innovations se diffusent d'abord le long du « vecteur » d'une tradition discursive, avant de se diffuser dans d'autres traditions discursives. Elle reçoit de cet ancrage diachronique des caractéristiques qui la rendent assez originale et peut-être un peu déroutante parmi d'autres notions qui sont plutôt, me semble-t-il, élaborées dans une perspective synchronique, et pourrait permettre d'enrichir et d'élargir d'autant la discussion sur les genres textuels.

La notion de tradition discursive insiste sur les relations de répétition et d'imitation entre les textes : les textes sont d'abord de larges répétitions et imitations de textes antérieurs. Schlieben-Lange (1996) parle de « la répétition, la récurrence, le riche réservoir où puisent les sujets producteurs de textes »; " la répétition, la récurrence d'un stock de formules et des passages tout faits »; Koch (1997) de «comportement communicatif typique et reproductible»; Aschenberg (2003) souligne qu'il n'y a pas de discours qui ne « [recycle] des modèles généraux »; Glessgen (2007: 104) enfin indique :

[Un] énoncé ne tient pas simplement compte du diasystème. Il reproduit en même temps des modèles préétablis pour une situation communicative donnée : s'il s'agit d'écrire une lettre officielle, les éléments d'ouverture et de salutation [...] voire le choix de la variété diasystématique sont définis d'avance. [...] Pour une lettre ou une conversation d'achat comme pour une poésie ou un texte scientifique, les énonciateurs reproduisent le modèle d'autres discours semblables appartenant au même genre textuel et ils puisent dans un vaste inventaire d'éléments de langue préfabriqués. La production de la parole représente alors plus un acte de reproduction de formes déjà existantes qu'un acte de libre création.

Les traditions discursives sont le résultat de ces relations d'imitation et de reprise. L'existence de proximités entre les textes est, dans cette perspective, un produit de l'histoire. Puisqu'elles résultent des relations de reprises entre les textes, les traditions discursives sont donc fondamentalement des «traditions », des fossilisations pourrait-on dire. Ceci n'implique pas bien sûr qu'elles n'aient pas également une histoire et qu'elles ne soient pas soumis au changement : " en tant que tradition discursive, les genres de texte sont caractérisés par leur dynamique et leur changement continu » (Wilhelm 2001). Cette notion prolonge en cela la notion de norme cosérienne (voir ci-dessous), c'est-à-dire la réalisation traditionnelle du système fonctionnel d'une langue, par opposition au système fonctionnel lui-même, qui contient les possibilités de changement. 
Dans ce cadre coserien les traditions discursives sont clairement distinguées des différents axes de variation (diastratique, diatopique, diaphasique) qui constituent le diasystème et en particulier de la variation diastratique. En effet, il existe des traditions discursives non marquées sur un plan diastratique ou mélangeant plusieurs variétés sur ce plan ( $c f$. Glessgen 2007). De plus, contrairement aux axes de variation du diasystème, les traditions discursives sont supra-linguistiques. Les mêmes traditions discursives peuvent en effet s'observer dans différentes langues : « [...] nous retrouvons, dans toute la Romania, les mêmes types de textes, les mêmes genres littéraires et non littéraires ou — comme je dirais — les mêmes traditions discursives qui accompagnent pour ainsi dire le passage à l'écrit. " (Koch 1993 : 41 ; cf. également 1997). C'est une tradition discursive particulièrement translinguistique qui est donnée comme exemple canonique chez plusieurs des auteurs (eg. Aschenberg 2003: 1) : celui de l'incipit des contes (il était une fois / once upon a time / es war einmal / erase una vez / ...). Cette indépendance des langues et des traditions discursives, voire la tendance à les mettre sur le même plan, est soulignée dans cette observation de Wilhelm (2001) :

Tout comme il ne peut y avoir d'expression linguistique « en dehors » d'une langue historique donnée - notre discours est nécessairement italien, français, etc. — de même on ne peut parler « en dehors » d'une tradition discursive déjà établie : notre parole utilise nécessairement la forme du salut, de la collection de poésies, de la conversation téléphonique, la lettre, etc.

Le terme de tradition discursive trahit bien sûr l'influence de la notion de « formation discursive » proposée par Foucault. Mais cette influence reste finalement assez secondaire ; en particulier, l'historicité des traditions discursives est réellement linguistique et se distingue donc, me semble-t-il, de l'histoire comme trace idéologique ( $c f$. ci-dessous).

Dans cette conceptualisation souple et adaptée à plusieurs points de vue sur le texte, deux critères prennent une place centrale (Kabatek : 2011) : celui de la pertinence épilinguistique - la tradition discursive est une catégorie pertinente pour les locuteurs - , et celui de la récurrence : pour établir une tradition discursive, il faut observer une forme de répétition. C'est une rencontre originale dans la mesure où les théories qui insistent sur la dimension de récurrence et a fortiori de fréquence abandonnent souvent la question de l'épilinguistique pour se concentrer sur le critère de l'homogénéité interne, statistique ; tandis que les théories qui insistent sur la dimension épilinguistique ne semblent pas le plus souvent considérer comme constituants les phénomènes de répétition.

La notion de tradition discursive est ainsi établie sur des critères assez minimaux et généraux, en un certain sens : celui de la répétition et de la traditionnalité. De fait, la plupart des auteurs voient d'abord ce terme comme un hyperonyme — ou du moins comme compatible avec — d'autres dénominations : Koch \& Esterreicher (2001 : 588) présentent ainsi les traditions discursives comme un « terme qui englobe les types de textes, les genres (littéraires et non-littéraires), les styles, etc., qui transcendent d'ailleurs les communautés linguistiques. »

De plus, cette perspective diachronique fait passer au second plan les questions d'inventaire et de typologie des textes qui se posent en synchronie : ce qui est pertinent, ce sont les liens d'un texte avec des textes antérieurs, les relations de reprise et d'imitation entre textes dans une tradition.

Enfin, dernier témoignage du caractère peu formel de cette notion, une tradition discursive peut se développer à n'importe quel niveau de la textualité : le discours 
médiatique, l'article de journal, l'article de quotidien, le titre de Libération; chacun de ces niveaux peut établir une traditionnalité et être considéré comme une tradition discursive (Kabatek 2011 : 92).

Le primat de la récurrence et du caractère formulaire permet, comme on le voit, de rapprocher un grand nombre de phénomènes, de la phraséologie au genre.

L'importance accordée aux « moules », aux éléments «préfabriqués », rapproche cette notion de celle de prefab (« [...] fluent and idiomatic control of a language rests to a considerable extent on knowledge of a body of "sentence stems" which are "institutionalized" or "lexicalized". ", Pawley \& Syder 1983 : 191).

Par cette insistance sur la répétition, la notion de tradition discursive contient une conceptualisation de la notion de fréquence textuelle et fournit un cadre d'interprétation des faits de fréquence, qui me paraît être l'un des enjeux les plus importants de cette notion; j'y reviendrai ci-dessous.

\section{La notion de tradition discursive parmi d'autres propositions théoriques}

Afin de mieux apprécier l'intérêt de cette notion je voudrais la comparer à d'autres notions proches, qui tentent également de rendre compte de l'existence de proximités entre textes. J'utiliserai le terme de « genre » comme hyperonyme pour ces notions («types de textes », « discours », « sous-langage », etc.)

Ces notions peuvent mettre en avant différents critères pour apprécier les proximités entre textes et fonder des typologies textuelles. On peut distinguer les cas de figure suivants (sans prétendre à l'exhaustivité) :

Le critère de l'homogénéité interne consiste à privilégier la proximité formelle entre des textes : les genres sont des classes de textes qui sont les plus similaires possible sur le plan de propriétés formellement identifiables (par exemple, de fréquences de traits morphosyntaxiques).

Le critère épilinguistique se base sur la pertinence, pour les locuteurs, des distinctions et des dénominations.

Le critère fonctionnel permet de regrouper les textes selon leur fonction (convaincre, informer, enseigner, divertir...)

Le critère du statut sémiotique consiste à privilégier ce qui permet de caractériser le genre comme forme sémiotique autonome, impliquant souvent un contrat de lecture et un certain nombre de contraintes interprétatives.

Le critère d'institution sociale fait privilégier le caractère socialement institué d'un genre - quand il est conçu principalement comme le résultat ou le vecteur d'un contenu idéologique, ou comme élément d'une praxéologie.

Enfin, on privilégie un critère psychologique quand on met en avant ses corrélats ou ses substrats psychologiques ou cognitifs.

\section{L'homogénéité formelle}

La plupart des notions de genre privilégient l'un ou l'autre de ces critères typologiques, parfois plusieurs. La notion de «type de textes » de Biber (1988) est un exemple du premier cas de figure, l'homogénéité interne. Les types de textes, chez cet auteur, sont en effet des ensembles de textes qui partagent les mêmes caractéristiques morphosyntaxiques. Dans cette perspective les genres sont d'abord des classes, des ensembles; ils ne peuvent être observés que dans un corpus et au 
moyen de méthodes statistiques pour synthétiser, dans chaque texte du corpus, les fréquences d'un grand nombre de phénomènes. La proximité formelle entre les textes est indépendante de toute pertinence épilinguistique. La dimension épilinguistique est prise en charge, chez Biber, par une autre notion, celle de «register ». La notion de type de texte, selon lui, est proprement linguistique (c'est-à-dire : formelle et indépendante du sentiment linguistique), tandis que la notion de register relève davantage du social.

Cette conception, ou d'autres reposant sur la même proximité formelle entre textes a reçu un large écho. D'une part, elle recèle une objectivité apparente qui permet de se prémunir contre les catégorisations perçues comme littéraires ou traditionnelles. D'autre part, elle se prête à une opérationnalisation concrète grâce aux méthodes factorielles ou les méthodes de classification qui peuvent être mises en œuvre relativement facilement. De nombreux auteurs ont souligné l'enjeu d'accéder à l'observation des régularités entre textes sur la base à la fois d'un grand nombre de textes et d'un grand nombre de propriétés empiriques. Gadet (2003 : 59) indique ainsi : «Cette perspective [il s'agit plus généralement des classifications statistiques], qui oblige à prendre en compte les énoncés selon des principes de différents ordres, devrait renouveler la définition des genres en les montrant comme des faisceaux de paramètres, et non plus des rubriques rhétoriques ou situationnelles héritées de la tradition »; Blanche-Benveniste (2005) remarque : «On peut enfin produire des analyses distributionnelles étendues à de vastes contextes, sur lesquels on peut mesurer l'effet des collocations entre prosodie, lexique et grammaire ». L'intérêt de ces méthodes ne se limite pas à la variation textuelle : elle peut s'appliquer également à la distinction des variétés du diasystème (« La distinction concrète des différentes variétés est plus le résultat d'une étude statistique qu'une réalité linguistique reconnue par les locuteurs », Glessgen 2007 : 102).

La limite de ces méthodes tient au caractère ad hoc de ces résultats (Loiseau 2010) : si on peut facilement mettre au jour, dans tout type de corpus, des regroupements de textes pertinents, ces regroupements ne paraissent pas permettre d'affiner la notion de genre ou de progresser vers des typologies généralisables au-delà du corpus considéré. Ces regroupements paraissent dus, selon les cas, à des caractéristiques variées (thématiques, modalités, paramètres variationnels, facteurs fonctionnels). On peut mobiliser ici la distinction rappelée par Rastier (2001) entre « identification » et « caractérisation » :

[...] distinguons [...] l'identification et la caractérisation, ou si l'on préfère les traits « morelliens » et les traits « spitzériens ». Morelli, médecin italien, révolutionna à la fin du XIX ${ }^{\mathrm{e}}$ siècle les attributions de tableaux en décelant des traits, notamment anatomiques, comme les lobes d'oreille, dont la facture caractéristique échappait jusque-là aux faussaires comme aux experts. Quant à Spitzer, on lui a maintes fois reproché de caractériser les œuvres par des traits formels qui paraissaient choisis arbitrairement, et lui permettaient pourtant d'entrer dans le cercle d'une interprétation révélatrice.

Les expériences de classification en types de textes relèvent en effet de l'« identification »: elles permettent avec une certaine sûreté de déterminer de quel ensemble un texte donné est le plus proche. De même que, dans des tableaux, des traits de facture auxquels on ne prête aucune attention peuvent devenir des signatures, de mêmes des proportions de traits morphosyntaxiques, à l'échelle d'un traits, échappent à l'attention consciente mais indexent impitoyablement un texte dans tel ou tel ensemble. Mais les expériences de classification ne permet- 
tent pas de décrire ce texte ni l'ensemble en question. Les faits quantitatifs utiles pour trancher une question d'appartenance d'un texte à un type de texte sont des «patrons » arbitraires qui n'intéressent pas nécessairement la description.

Ce n'est sans doute pas un hasard si les travaux d'attribution d'auteur ont été les premiers à utiliser des données quantitatives : dans cette perspective, l'objectif d'attribuer le texte à un auteur se satisfait d'une "signature » trahissant la pâte d'un auteur, d'autant plus pertinente comme signature et «tatouage » de l'œuvre que, précisément, personne n'aura songé à l'imiter puisqu'elle n'a pas de pertinence interprétative. Les traits qui permettent d'identifier un sonnet comme shakespearien ne sont pas nécessairement ceux qui intéressent l'interprétation ou l'imitation de Shakespeare. Comme les «traits spitzériens », les faits quantitatifs utiles à la description, au contraire, doivent pouvoir être interprétés, c'est-à-dire rapportés à d'autres catégories descriptives.

Soulignons que le critère typologique de l'homogénéité formelle n'est pas nécessairement associé à des méthodes statistiques. On pourrait rapprocher de ce type de typologie les langues de spécialité ou les sous-langages de Harris. Dans le premier cas, il s'agit principalement de lexique partagé ( Quant aux langues de spécialité, les spécialistes semblent d'accord sur le point que, en dehors du lexique, il existe peu de traits particuliers qui ne s'expliqueraient pas mieux par le genre textuel »; Dufter \& Stark 2002 : 86) ; dans le second, de constructions.

On peut également rapprocher de cette perspective le projet de « grammaire de discours » de Sueur (1982) : «J'appellerai « grammaire de discours » l'étude des régularités dans la distribution, les uns par rapport aux autres, des faits de lexique, de syntaxe, et d'énonciation dans un corpus. »

\section{Le sentiment épilinguistique}

De nombreux auteurs soulignent l'importance de prendre compte la pertinence, pour les locuteurs, des genres distingués dans une typologie. Branca-Rosoff (1999: 5-6) souligne par exemple cette importance de l'épilinguistique, « [toujours] présent dans la constitution des genres de l'analyse. » (cf. également Loureda Lamas 2004). Le critère du sentiment linguistique est souvent opposé au critère de l'homogénéité formelle : «Prendre en compte un répertoire historique de genres, ce n'est pas faire l'hypothèse de l'homogénéité de chaque genre mais seulement de l'intérêt de son caractère d'observatoire. À partir de données configurées en genres, on peut observer l'émergence des outils linguistiques que construisent les locuteurs dans des pratiques sociales réelles et bien délimitées. » (Branca-Rosoff 1999).

Il peut être intéressant à nouveau de comparer les typologies textuelles avec la description de la variation. Il a déjà été noté que les phénomènes qui sont «agglomérés » pour identifier des variétés sur le critère de l'homogénéité interne n'ont pas tous le même statut épilinguistique. Gadet (2003) indique : «Ainsi, on peut supposer que les variables qui permettent d'évaluer ou d'affirmer la différence entre ingroup et outgroup ont une saillance qui les rend pour le locuteur socialement signifiantes. Loin que la fréquence soit toujours significative, une seule occurrence de formes fortement stigmatisées peut suffire a connoter négativement un discours (pour le français, on peut penser a des «fautes» de morphologie, comme des chevals, il as 'agi ou il éteignera, ainsi qu'à certaines liaisons fautives). » (cf. Loiseau $2010: 25)$. Selon Dufter \& Stark (2002:83) il faut « [...] [considérer] une telle identifiabilité (des variétés) par les non-linguistes comme condition d'existence indispensable pour toute variété. » 


\section{La dimension sociale et praxéologique}

Dans la catégorie des notions de genre qui mobilisent principalement un critère praxéologique il me semble qu'on peut ranger, au risque de faire une cote mal taillée, un grand nombre de notions assez différentes mais qui relèvent de l'analyse du discours et, plus généralement, de la prise en compte du social. Il s'agit des notions comme celles issues de la notion de formation discursive ( $c f$. Cambon \& Léglise 2008), de discours chez Rastier, qui insistent sur la dimension praxéologique, c'est-à-dire sur le fait que les pratiques langagières sont des pratiques sociales et que ce qui structure et différencie les pratiques langagières ce sont des déterminations venues du social (ou du discours en tant qu'il s'agit d'un fait social). Dans ces perspectives il est important de « [prendre] en compte la situation pratique au sein de laquelle se déroulent les échanges langagiers afin de les caractériser. » (Cambon \& Léglise $2008: 3$ ), de décrire les effets de sens « en tant que produit par les effets sociaux. » (Cambon \& Léglise 2008 : 5) À l'intérieur de cette catégorie il faudrait naturellement distinguer des sous-ensembles selon que l'on met en avant le dispositif énonciatif, des visées praxéologiques, le caractère d'institution sociale ou des contenus idéologiques.

Dans cette perspective à nouveau il y a une tension entre les indications typologiques des critères praxéologiques et les regroupements sur des bases formelles. Sonia Branca-Rosoff (1999) indique ainsi : "L'instabilité de la relation entre formes et comportements sociaux institutionnalisés est une difficulté centrale pour toute définition a priori des genres. Une conception historique qui défige les catégories ou qui n'y voit que des entités provisoirement stabilisées permet de traiter une partie des écarts constatés. »

Les typologies qui sont basées sur des critères énonciatifs à la suite de Benveniste et de la distinction entre histoire et discours (e.g. Simonin-Grumbach 1975) peuvent être rattachées à cette catégorie dans la mesure où les configurations identifiées seront analysées le plus souvent en termes de positionnements idéologiques (Charolles \& Combettes 1999 : 81).

\section{Le critère sémiotique}

Je rattache à ce critère les typologies qui identifient chaque genre à un « contrat de lecture ». C'est 1'une des caractéristiques de la notion de genre proposée par Rastier : le genre, chez cet auteur, est une forme sémiotique (au sens où elle règle le rapport entre plan de l'expression et du contenu) : " [un genre] détermine ce mode de corrélation entre plan du signifiant et plan du signifié que l'on peut nommer sémiosis textuelle» (Rastier 2001b). Cette «sémiosis textuelle » est associée à des régime génétique, mimétique et herméneutique, c'est-à-dire, d'une certaine façon, des contrats de lecture. Ce qui caractérise cette élaboration de la notion de genre est le fait qu'il s'agit d'une unité sémiotique et linguistique de plein droit.

\section{Le critère psychologique}

Parmi les notions de genre qui illustrent l'accent sur la composante psychologique on peut citer les travaux de Bronckart (1997 : 138) : "A l'échelle d'un agent singulier, la production d'un nouveau texte empirique [doit] être conçue comme le résultat de la mise en interface entre les représentations construites par l'agent à propos de sa situation d'action (motifs, intentions, contenu thématique à transmet- 
tre, etc.), et les représentations de ce même agent concernant les genres disponibles dans l'intertexte. [...] Dans cette optique, tout nouveau texte empirique [est] donc nécessairement construit sur le modèle d'un genre, [...] il rel[ève] d'un genre. »

\section{Les critères fonctionnels}

Les catégorisations fonctionnelles ont été développées par exemple par Adam (2001) qui distingue cinq «types de textes » : texte narratif, descriptif, explicatif, argumentatif et dialogal.

\section{Bilan}

Cette classification, ainsi que la répartition des notions mentionnées entre ces différentes perspectives typologiques, peut sans doute être très largement affinée, mais l'identification des critères typologiques me semble éclairer les différences entre les notions de genre. Les différentes notions de genres privilégient chacune, en particulier, un de ces critères typologiques. Elles sont cependant rarement «pures » : par exemple, les «types de textes » de Biber relèvent principalement d'une approche fondée sur l'homogénéité formelle ; cependant, les classes produites sont interprétées dans des termes plutôt fonctionnels. La notion de genre proposée par Rastier mobilise également un aspect praxéologique (les genres sont « articulées aux pratiques sociales », 2001b) ; un critère d'homogénéité formelle (« un genre se définit [...] par la cohésion d'un faisceau de critère. », 2001b. Ces critères sont cependant trop abstraits — il s'agit de la thématique, de l'univers actantiel... cf. Rastier 2001 - pour être assimilables aux marques utilisées dans les typologies formelles mentionnées plus haut.).

Les traditions discursives, si elles relèvent principalement d'un critère diachronique, intègrent également des aspects « contrat de lecture »- comme du reste plusieurs autres propositions : Koch \& Esterreicher $(2001: 602)$ associent aux traditions discursives des «schémas de production et de réception ». Wilhelm (2001: 467) associe une perspective fonctionnelle, secondaire, aux traditions discursives.

À l'inverse, plusieurs notions de genre mentionnent un critère diachronique. Adam parle ainsi de «tradition», Rastier de «lignée générique »; la notion de formation discursive et ses dérivées accordent naturellement une importance particulière à l'histoire ; celle-ci est présente en particulier dans la notion de « mémoire discursive ${ }^{(1)}$. Courtine (1981:52-53) la définit ainsi :

Ce que nous entendons par le terme de « mémoire discursive » est distinct de toute mémorisation psychologique du type de celle dont les psycholinguistes s'attachent à produire la mesure chronométrique [...]. La notion de mémoire discursive concerne l'existence historique de l'énoncé au sein de pratiques discursives réglées par des appareils idéologiques, elle vise ce que Foucault $(71$, p. 24) relève à propos des discours religieux, juridiques, littéraires, scientifiques, « discours qui sont à l'origine d'un certain nombre d'actes nouveaux, de paroles qui les reprennent, les transforment, ou parlent d'eux, bref, les discours qui indéfiniment, pardelà leur formulation, sont dits, restent dits et sont encore à dire ».

Il me semble cependant que l'histoire ou la mémoire dans les élaborations is-

(1) Je remercie Marie-Anne Paveau de m'avoir signalé cette référence. 
sues de la notion de formation discursive doivent être distinguées de la diachronie et de l'histoire au sens linguistique ; elle concerne davantage l'hypothétique réification dans les signes linguistiques de faits non linguistiques que le changement des formes linguistiques elles-mêmes.

\section{Un enjeu des traditions discursives : l'interprétation des faits de fréquence}

Je voudrais insister dans cette dernière partie sur ce qui me semble être l'un des enjeux de la notion de tradition discursive, à savoir le fait qu'elle permet de mieux expliquer les faits de fréquences linguistiques. Je reprends et prolonge ici des raisonnements esquissés dans (Loiseau 2010).

Afin d'apprécier cet aspect des traditions discursives il est intéressant de revenir, d'abord, sur la notion de norme de Coseriu à laquelle la notion de traditions discursives est fortement liée : les deux notions relèvent toutes deux de la dimension de traditionnalité (par opposition à la créativité) de la langue.

La norme au sens de Coseriu (1982) est la réalisation traditionnelle du système fonctionnel (de la langue). Celle-ci est définie comme « tout ce qui, dans la 'technique du discours', n'est pas nécessairement fonctionnel (distinctif), mais qui est tout de même traditionnellement (socialement) fixé, qui est usage commun et courant de la communauté linguistique. Le système, par contre, comprend tout ce qui est objectivement fonctionnel (distinctif). »(Coseriu 2001 : 205). Elle peut spécifier ce que le système laisse indéterminé. Prenons l'exemple de la neutralisation en finale de l'opposition entre sourdes et sonores en russe. Puisque l'opposition est neutralisée, la réalisation peut être sourde ou sonore sans conséquence fonctionnelle ; cependant elle est toujours réalisée sous la forme sourde : c'est un fait de norme.

La norme peut rendre compte de variantes : les trois degrés d'abstraction que sont la langue (le système fonctionnel), la norme et le discours s'ordonnent selon le nombre d'unités qu'ils font distinguer. En espagnol par exemple, on décrit un phonème /o/, deux types de [o] du point de vue de la norme (« deux variantes typiques »), et bien sûr une infinité de réalisations dans le discours (1982: 72).

Enfin, et c'est le point qui nous intéresse ici, les faits de normes relèvent d'aspects fréquentiels ; par exemple, la fréquence d'un phonème peut être " anormale » (1982: 71) : «El fonema /x/ (en la grafía corriente j, o g, delante de e, i) es un elemento común del sistema fonológico español; sin embargo, una frase como Artajo trajo la valija abajo produce un extraño efecto "estilístico", porque la frecuencia relativa del fonema es mucho menor en la norma española. »

Le concept de norme tel qu'élaboré par Coseriu, qui insiste sur la traditionnalité de l'activité de parler concrète, intermédiaire entre la parole et le système fonctionnel, permet particulièrement de prendre en compte les propriétés de fréquence, de récurrence, ou de répétition des phénomènes linguistiques. La norme est ainsi faite d'éléments « no accesorias y esporádicas » (1982:89); « simple tradición constante, elemento común » $(1982: 96)$; «normal, repetido en una comunidad » (1982: 57) ; «normales y repetidos en el hablar de una comunidad» (1982: 55); « elementos normales y constantes » (1982:69); " repetición de un modelo anterior » (1982: 57 et passim ) ; «traditionnel et “usuel” » (1987:53-54), etc. Comme dans toutes les sciences humaines, la normativité (toujours au sens de 
Coseriu et non au sens de prescriptions) se traduit quantitativement par des fréquences remarquables. Les faits normaux sont des faits fréquents.

On voit que les traditions discursives étendent les acquis de la norme dans un cadre textuel : là aussi, la traditionnalité est fondamentale et s'observe à travers des faits de fréquences textuelles.

On peut le comparer aux cadres cognitifs contemporains (notamment « usagebased ») qui insistent également sur les phénomènes de fréquences, mais du point de vue de leurs effets cognitifs. Les grammaires usage-based proposent également d'intégrer une dimension " première », constitutive, de la répétition. Comme dans la perspective cosérienne, l'accent est mis sur l'activité de reconfiguration du système dans l'activité de parler; Bybee \& Hopper (2001:2) citent d'ailleurs Coseriu à l'appui de l'idée que « The fixing of linguistic groups of all kinds as recognizably structural units (word and phrase units) is an ongoing process; it is the result at any point in time of the "constant resystematization" of language. (Coseriu 1954)»

La dimension de répétition et la prise en compte de la fréquence sont dans ce cadre rapportées à l'activité cognitive : si la répétition importe, c'est à travers l'hypothèse que ses effets cognitifs rendent compte de la stabilisation et de la systématisation du système (Schmid $2007: 119$ ) : «The frequency of occurrence of concepts or constructions in a speech community has an effect on the frequency with which its members are exposed to them. The (tacit rather than explicit) implication is that this results in some kind of collective automatization effect, which makes it possible to talk of the degree of entrenchment of a concept or construction in a given language. »

Ainsi la fréquence détermine les unités pertinentes de l'analyse, en fonction d'hypothèses sur le «stockage cognitif» des unités : « High-frequency words and phrases grow strong with repetition and loom large, forming looser connections with other items, while low-frequency words and expressions are less prominent but grain stability by conforming to patterns used by other items. \(Bybee $2007: 9)$

Plusieurs différences avec le cadre cosérien méritent d'être soulignées. On observe une alternative entre deux modèles de prise en charge de la fréquence : dans le cas de Coseriu, la fréquence est rapportée à un plan d'analyse historique, tandis que dans le cas des grammaires usage-based, elle est rapportée à un plan cognitif.

L'opposition porte aussi sur le statut empirique de la fréquence : elle est directement observable dans le premier cas, mais non dans le second, puisque la fréquence qui compte est celle qu' « enregistre » l'activité cognitive, dont le fonctionnement demeure évidemment largement obscur. Les catégories descriptives, dans ce cadre, sont gagées sur les hypothèses relatives au fonctionnement cognitif : " The general claim is that this more frequent sequence gradually moved away from its source construction becoming more autonomous. » (Bybee 2006 : 11)

L'opposition porte enfin sur le régime interprétatif dont relèvent les deux conceptions de la fréquence. En tant que forme historique, les normes relèvent partiellement d'une interprétation et d'une herméneutique. Dans le second cas, la fréquence relève d'une explication de type causal. La fréquence sert alors au passage depuis les phénomènes observables vers un arrière-plan cognitif. La répétition se traduit en faits cognitifs qui ne sont pas eux-mêmes directement observables. Alors que dans le cadre d'une théorie de la norme la fréquence est quelque chose 
d'essentiellement empirique et historique, dans un cadre cognitif la fréquence relève de phénomènes cognitifs universels.

Le recours au fonctionnement cognitif comme lieu d'organisation des catégories de la description fait retrouver un paradoxe récurrent lié à la fréquence : elle est à la fois empirique et non directement observable. Ici, il s'agit plutôt d'une fréquence universelle, que d'une fréquence historique et située : les principes de l'entrenchment sont universels et indifférents aux langues et aux situations historiques, tandis que la norme n'a aucune réalité hors d'une configuration historique donnée. De plus, la «localisation » cognitive de la fréquence oblige à ne pas distinguer entre différents types de normes (normes "culturelles », diasystméiques, idiolectales, textuelles, etc.), à l'origine des régularités observables, à faire de la fréquence un tout indécomposable.

Ces deux propositions d'articulation de la langue et de l'activité de parole concrète sensibles à la dimension de fréquence s'opposent donc comme une perspective universaliste et naturalisante à une perspective historique sur les faits de fréquence.

Parole, norme et langue peuvent être vus comme correspondant à trois types d'historicité, et rapportés aux trois types d'historicité distingués dans (Kabatek 2005 : 151 sqq.). Le premier type d'historicité correspond à la langue : « La langue en tant que langue particulière est l'histoire d'une communauté intériorisée dans l'individu. » (2005: 151). Le second type d'historicité correspond à la dimension de répétition et correspond peut-être particulièrement au niveau de la norme (2005:152) :

Le second type d'historicité, en revanche, concerne tout type de phénomène culturel, voire langagier. Cela implique les traditions au sein d'une communauté, la création récurrente d'objets culturels à partir de certaines similarités ou de l'identité partielle de phénomènes culturels antécédents. Il s'agit donc d'objets culturels dont l'actualisation, qui ne correspond jamais exactement à la tradition, est disponible à la communauté. La langue en tant qu'objet se manifeste par des textes qui se rapportent à la tradition par la répétabilité d'une certaine finalité textuelle et surtout par certains caractères formels. La répétabilité de formes textuelles comprend une échelle continuelle de marquage depuis la tradition minimale - p. ex. une certaine dénomination de texte ou une formule dans un texte qui par ailleurs n'est pas fixé par la tradition —, à l'organisation formelle et le figement définitif d'un texte.

Enfin le troisième type d'historicité concerne « les phénomènes individuels, non reproductibles, c'est-à-dire le texte en tant qu'individu, puisque chaque texte exprimé peut être situé historiquement. » (2005: 153).

En conclusion, on voit que la notion de tradition discursive peut être vue comme une extension de la norme au niveau textuel, puisque toute deux s'attachent à rendre compte de phénomène de répétition; la norme reste cependant une réalisation d'un système fonctionnel (d'une langue), tandis que la tradition discursive n'est pas attachée à une langue ni a un diasystème. Comme la norme, la notion de tradition discursive fournit un cadre théorique intéressant pour interpréter les fréquences textuelles; en comparant ce cadre théorique aux développements des grammaires usage-based, on voit que la fréquence est construite, dans le premier, comme un fait historique et linguistique, alors qu'elle est principiellement rapportée, dans les seconds, à des universaux cognitifs trans-linguistiques. 


\section{Conclusion}

La notion de tradition discursive est une notion originale, issue de travaux en histoire de la langue, mais qui intéresse et interroge plus généralement les travaux en typologie textuelle et les notions qui tentent de rendre compte de l'appartenance des textes à des patrons plus généraux.

Deux notions sont mises en avant dans l'élaboration de cette notion : la récurrence et la pertinence épilinguistique. Cette association est relativement originale et est rendue possible, me semble-t-il, par la perspective diachronique et l'importance accordée à la traditionnalité et à l'imitation. Différents phénomènes rarement rapprochés, et qui vont de la phraséologie aux genres, se trouvent rapprochés dans cette perspective qui met l'accent sur la traditionnalité et les différents types d'historicités linguistiques.

L'un des enjeux de cette notion est de proposer un cadre riche et stimulant pour mieux comprendre la fréquence textuelle. Elle ouvre des perspectives méthodologiques pour l'interprétation des faits de fréquences en corpus.

\section{Bibliographie}

ADAM, J.-M. (2001 [1997]) : Les textes, types et prototypes -Récit, description, argumentation, explication et dialogue, Paris : Nathan.

A SCHENBERG, H. (2003) : «Diskurstraditionen - Orientation und Fragestellungen », in Heidi Aschenberg et Raymund Wilhelm (éd.), Romanische Sprachgeschichte und Diskurstraditionen, Tübingen : Gunter Narr Verlag Tübingen, 118.

Douglas, B. (1988) : Variation across Speech and Writing, Cambridge, Cambridge University Press.

BLANCHE-BENVENISTE, C., (2005) : «L'Étude grammaticale des corpus de langue parlée en français », Geoffrey Williams (éd.), Rennes : Presses universitaires de Rennes, 47-66.

BRANCA-RosofF, S., (1999) : «Types, modes et genres : entre langue et discours », Langage et société, 87, 5-24, http://www.cavi.univ-paris3.fr/ilpga/ED/dr/drsb/sb-pdf/intro-Branca-LS87.pdf.

- (1999b) : «Des innovations et des fonctionnements de langue rapportés à des genres », Langage et société, 87, 115-129, http://www.cavi.univ-paris3.fr/Ilpga/ed/dr/drsb/sb-pdf/innovations-Sonia-LS87.pdf.

BRONCKART, J.-P. (1997) : Activité langagière, textes et discours, Lausanne : Delachaux \& Niestlé.

BYBeE, J. L., \& HopPeR, P. (2001) : «Introduction », in Bybee, Joan L. \& Hopper, P. (éd.), Frequency and the Emergence of Linguistic Structure, Amsterdam : John Benjamins. 
Bybee, J. L., (2007) : Frequency of Use and Organization of Language, Oxford : Oxford University Press.

- (2006) : «From Usage to Grammar : The Mind's Response to Repetition », Language, 4, 82, 711-733.

Charolles, M. (1976) : «Grammaire de texte, théorie du discours, narrativité », Pratiques, 11-12, 133-154.

Charolles, M. \& Combettes, B. (1999) : «Contribution pour une histoire récente de l'analyse du discours » Langue française, 121, 76-116.

CAMBON, E. \& LÉGLISE, I., (2008) : « Pratiques Langagières et registres discursifs. Interrogation de deux cadres en sociologie du langage », Langage et Société, 124, 15-38.

COSERIU, E., (2001) : L'homme et son langage, H. Dupuy-Engelhardt, J.-P. Durafour et F. Rastier (éd). Leuven : Peeters.

— (1982 [1952]) : «Sistema, norma y habla », in Teori'a del lenguaje y lingui'stica general, Madrid: Gredos, 11-113.

COURTINE, J.-J., (1981) : «Quelques problèmes théoriques et méthodologiques en analyse du discours. À propos du discours communiste adressé aux chrétiens. » Langages, 62, « Analyse du discours politique », 9-128.

DUFTER, A. \& STARK, E., (2002) : «La variété des variétés : combien de dimensions pour la description ? », Romanistisches Jahrbuch, n53, 81-108.

GÉVAUDAN, P. (2009) : «Grammaticalité et traditions discursives de l'énonciation polyphonique », in Alexandra Kratschmer, Merete Birkelund \& Rita Therkelsen (éd.), La polyphonie : outil heuristique linguistique, littéraire et culturel, Berlin : Frank \& Timme, 99-124.

Glessgen, M.-D., (2007) : Linguistique romane, Paris : Armand Colin.

HABERT, B. (1983) : «Etude des formes spécifiques et typologie des énoncés (les résolutions générales des congrès de la CFTC-CFDT de 1945 à 1979) », Mots, 7, 97-124.

KABATEK, J. (2001) : « ¿Cómo investigar las tradiciones discursivas medievales ? El ejemplo de los textos jurídicos castellanos », dans Lengua medieval y tradiciones discursivas en la Península iberíca : descripción gramamtical pragmática histórica - metodología, Daniel Jacob \& Johannes Kabatek (éd.), Madrid : Iberoamericana/Vervuert, 97-132.

- (2004) : «Tradiciones discursivas jurídicas y elaboracíon lingüística en la España medieval », Cahiers de Linguistique et de Civilisation Hispaniques Médiévales, 27, 249-261, http://www.persee.fr/web/revues/home/prescript/article/cehm_0396-9045_2004_num_27_1_1623.

- (2005) : «À propos de l'historicité des textes », in Murguía A. (éd.) Sens et référence, Mélanges pour Georges Kleiber, Tübingen : Gunter Narr : 149158.

- (2008) : Sintaxis histórica del español y cambio lingüístico-Nuevas perspectivas desde las Tradiciones Discursivas (Madrid/Frankfurt am Main : Iberoamericana/Vervuert).

— (2011) : «Diskurstraditionen und Genres » in Sarah Dessì Schmid, Ulrich Detges, Paul Gévaudan \& Wiltrud Mihatsch (éd.), Rahmen des Sprechens : Beiträge zu Valenztheorie, Varietätenlinguistik, Kreolistik, kognitiver und historischer Semantik; Peter Koch zum 60. Geburtstag, Tübingen : Narr, 89100.

KoCH, P. (1997) : « Diskurstraditionen : zu ihrem sprachtheoretischen Status und 
ihrer Dynamik », in Barbara Frank, Thomas Haye, \& Doris Tophinke (éd.), Gattungen mittelalterlicher Schriftlichkeit, Tübingen : Narr, 43-79.

- (1997) : «Diskurstraditionen : zu ihrem sprachtheoretischen Status und ihrer Dynamik », éd in Barbara Frank, Thomas Haye, \& Doris Tophinke Gattungen mittelalterlicher Schriftlichkeit, Tübingen : Narr, 43-79.

- (2008) : «Tradiciones discursivas y cambio lingüístico : el ejemplo del tratamiento vuestra merced en español », dans Sintaxis histórica del españoly cambio lingüístico, in Johannes Kabatek (éd.), Madrid/Frankfurt am Main : Iberoamericana/Vervuert, 53-87.

KOCH, P. \& ESTERREICHER, W., (2001) : «Gesprochene Sprache und geschriebene Sprache », in Günter Holtus, Michael Metzeltin, \& Christian Schmitt (éd.), Lexikon der Romanistischen Linguistik, vol. 2, Tübingen : Max Niemeyer Verlag, 584-627.

KoCH, P. \& SElig, M. (1993) : «Pour une typologie conceptionnelle et médiale des plus anciens documents/monuments des langues romanes ", in Selig M. (éd.) Le Passage à l'écrit des langues romanes, Tübingen : Narr, 39-82.

Loiseau, S. (2010) : «Les paradoxes de la fréquence », Energeia, 2, 20-55.

Loureda LAMAS, Ó., (2004) : « Pasado, Presente Y Futuro De La Tipología Textual. », in Gerda Haßler \& Gesina Volkmann (éd.), Geschichte Der Sprachwissenschaft in Texten Und Konzeptionen. Münster : Nodus Publikationen.

PAwley, A. \& Syder, F. H., (1983) : « Two Puzzles for Linguistic Theory : Nativelike Selection and Nativelike Fluency. ", in J.C. Richards \& R.W. Schmidt (éd.), Language and Communication, London : Longman.

RASTIER, F. (2001) : «Vers une linguistique des styles », L'information grammaticale, 89, 3-6.

- (2001b) : «Éléments de théorie des genres », Texto !.

— (2009) : «Préface » à la troisième édition de Sémantique interprétative, Paris : PUF.

SCHLIEBEn-LANGE, B. (1996) : Idéologie, révolution et uniformité de la langue, Paris : Mardaga.

- (1998) : «Les hypercorrectismes de la scripturalité », Cahiers de Linguistique Française, 15, 255-273, http://clf.unige.ch/num.php ?numero=20.

- (1983) : Traditionen des Sprechens, Stuttgart : Kohlhammer.

SCHMID, H.-J. (2007) : «Entrenchment, Salience, and Basic Levels » in Dirk Geeraerts \& Hubert Cuyckens (éd.), The Oxford Handbook of Cognitive Linguistics, Oxford : Oxford University Press.

SIMONIN-GRUMBACH, J. (1975) : «Pour une typologie des discours », in Julia Kristeva, Jean-Claude Milner, Nicolas Ruwet (dir.), Langue, discours, société, Pour Émile Benveniste, Paris : Seuil, 85-121.

SuEUR, J.-P., (1982) : «Pour une grammaire du discours », Mots, 5, 143-185.

Wilhelm, R. (2001) : «Diskurstraditionen », in Martin Haspelmath et al. (éd.), Language Typology and Language Universals (Handbücher zur Sprach- und Kommunikationswissenschaft), Berlin-New York : Walter de Gruyter, 467477. 